\title{
On some Hermite-Hadamard type inequalities for strongly $s$-convex functions
}

\author{
Yusuf Erdem ${ }^{1}$, Hasan Ogunmez ${ }^{1}$, Huseyin Budak ${ }^{2}$ \\ ${ }^{1}$ Department of Mathematics, Faculty of Science and Arts, Afyon Kocatepe Universtiy, Afyonkarahisar, Turkey \\ ${ }^{2}$ Department of Mathematics, Faculty of Science and Arts, Duzce University, Duzce, Turkey
}

Received: 13 December 2016, Accepted: 27 February 2017

Published online: 25 August 2017.

\begin{abstract}
In this paper, we establish some new results related to the left-hand of the Hermite-Hadamard type inequalities for the class of functions whose second derivatives are strongly $s$-convex functions in the second sense. Some previous results are also recaptured as a special case.
\end{abstract}

Keywords: Hermite-Hadamard type inequalities, Hölder inequality, strongly s-convex functions.

\section{Introduction}

The following definition is well-known in the literature a functions $f: I \rightarrow \mathbb{R}, \varnothing \neq I \subset \mathbb{R}$, is said to be convex on $\mathrm{I}$ if the inequality

$$
f(t x+(1-t) y) \leq t f(x)+(1-t) f(y)
$$

holds for all $x . y \in I$ and $t \in[0,1]$.

Theorem 1. [10] Let $f: I \subseteq R \rightarrow R$ be a convex function on the interval I of real numbers and $a, b \in I$ with $a<b$. If $f$ is a convex function then the following double inequality, which is well known in the literature as the Hermite-Hadamard inequality, holds

$$
f\left(\frac{a+b}{2}\right) \leq \frac{1}{b-a} \int_{a}^{b} f(x) d x \leq \frac{f(a)+f(b)}{2}
$$

In [5], ,Hudzik and Maligranda considered the class of functions which are s-convex in the second sense. This class is defined in the following way: a funciton $f:[0, \infty] \rightarrow \mathbb{R}$ is said to be s-convex in the second sense if

$$
f(t x+(1-t) y) \leq t^{s} f(x)+(1-t)^{s} f(y)
$$

holds for $x, y \in[0, \infty], t \in[0,1]$ and for some fixed $s \in[0,1]$. This class of s-convex functions in the second sense is usually by $K_{S}^{2}$.

It can be easily see that for $s=1 \mathrm{~s}$-convexity reduces to the ordinary convexity of functions defined on $[0, \infty)$. 
Inequality (2) has studied a huge amount of interest over the years. We just remember the recent studies in ([2]-[4],[9],[13],[15]).

Definition 1. [11] A function $f: I \rightarrow \mathbb{R}$ is called strongly s-convex with modulus $c$ if

$$
f(t x+(1-t) y) \leq t^{s} f(x)+(1-t)^{s} f(y)-c t(1-t)(b-a)^{2} .
$$

In [1], Angulo et al. proved the following Hermite-Hadamard type inequality for strongly $h$-convex function:

Theorem 2. Let $h:(0,1) \rightarrow(0, \infty)$ be a given function. If a function $f: I \subset \mathbb{R} \rightarrow \mathbb{R}$ is Lebesgue integrable and strongly h-convex with modulus $c>0$, then

$$
\frac{1}{h\left(\frac{1}{2}\right)}\left[f\left(\frac{a+b}{2}\right)+\frac{c}{12}(b-a)^{2}\right] \leq \frac{1}{b-a} \int_{a}^{b} f(x) d x \leq(f(a)+f(b)) \int_{0}^{1} h(t) d t-\frac{c}{6}(b-a)^{2}
$$

for all $a, b \in I, a<b$.

Corollary 1. Suppose that $f:[0, \infty] \rightarrow \mathbb{R}$ is a strongly $s$-convex function in the second sense with modulus $c>0$, where $s \in(0,1)$ (i.e $h(t)=t^{s}$ in (5)), then following inequalities hold;

$$
2^{s-1}\left[f\left(\frac{a+b}{2}\right)+\frac{c}{12}(b-a)^{2}\right] \leq \frac{1}{b-a} \int_{a}^{b} f(x) d x \leq \frac{f(a)+f(b)}{s+1}-\frac{c}{6}(b-a)^{2} .
$$

For more information and recent developments on inequalities for srongly convex function, please refer to ([1],[6],[7],[8],[12],[14],[16],[17]).

The aim of the paper is to establish some new Hermite-Hadamard inequalities for function whose second derivatives in absolute value are strongly $s$-convex.

\section{Main results}

To prove our main results, we consider the following Lemma given by Ozdemir et al. in [9].

Lemma 1. Let $f: I \rightarrow \mathbb{R}$ be a differantiable mapping on $I^{0}$ where $a, b \in I$ with $a<b$. If $f^{\prime \prime} \in L[a, b]$, then the following equality holds

$$
\frac{1}{b-a} \int_{a}^{b} f(x) d x-f\left(\frac{a+b}{2}\right)=\frac{(b-a)^{2}}{16}\left(\int_{0}^{1} t^{2}\left(f^{\prime \prime} t \frac{a+b}{2}+(1-t) a\right) d t+\int_{0}^{1}(1-t)^{2} f^{\prime \prime}\left(t b+(1-t) \frac{a+b}{2}\right) d\right) t .
$$

Theorem 3. Suppose that $f: I \subset[0, \infty] \rightarrow \mathbb{R}$ be a twice differantiable mapping on $I^{0}$ such that $f^{\prime \prime} \in L([a, b])$, where a,b $\in I$ with $a<b$. If $\left|f^{\prime \prime}\right|$ is strongly s-convex on $[a, b]$, for some $s \in(0,1]$ with modulus $c>0$, thenfollowing inequalities hold

$$
\begin{aligned}
& \left|f\left(\frac{a+b}{2}\right)-\frac{1}{b-a} \int_{a}^{b} f(x) d x\right| \leq \frac{(b-a)^{2}}{8(s+1)(s+2)(s+3)}\left\{\left|f^{\prime \prime}(a)\right|+(s+1)(s+2)\left|f^{\prime \prime}\left(\frac{a+b}{2}\right)\right|+\left|f^{\prime \prime}(b)\right|\right\}-\frac{c}{160}(b-a)^{2} \\
& \leq \frac{(b-a)^{2}}{8[(s+1)(s+2)(s+3)]} \times\left\{\left[1+(s+2) 2^{1-s}\right]\left[\left|f^{\prime \prime}(a)\right|+\left|f^{\prime \prime}(b)\right|\right]-\frac{\left[1+(s+1)(s+2) 2^{1-s}\right] c}{12}(b-a)^{2}\right\}-\frac{c}{160}(b-a)^{2} .
\end{aligned}
$$


Proof. Taking modulus on both sides of (7), we have

$$
\left|f\left(\frac{a+b}{2}\right)-\frac{1}{b-a} \int_{a}^{b} f(x) d x\right| \leq \frac{(b-a)^{2}}{16} \int_{0}^{1} t^{2}\left|f^{\prime \prime}\left(t \frac{a+b}{2}+(1-t) a\right)\right| d t+\frac{(b-a)^{2}}{16} \int_{0}^{1}(1-t)^{2}\left|f^{\prime \prime}\left(t b+(1-t) \frac{a+b}{2}\right)\right| d t .
$$

Since $\left|f^{\prime \prime}\right|$ is strongly s-convex, we get

$$
\left|f^{\prime \prime}\left(t \frac{a+b}{2}+(1-t) a\right)\right| \leq t^{s}\left|f^{\prime \prime}\left(\frac{a+b}{2}\right)\right|+(1-t)^{s}\left|f^{\prime \prime}(a)\right|-c t(1-t)(b-a)^{2}
$$

and

$$
\left|f^{\prime \prime}\left(t b+(1-t) \frac{a+b}{2}\right)\right| \leq t^{s}\left|f^{\prime \prime}(b)\right|+(1-t)^{s}\left|f^{\prime \prime}\left(\frac{a+b}{2}\right)\right|-c t(1-t)(b-a)^{2} .
$$

If we substitute the inequalities (10) and (11) in (9), then we obtain

$$
\begin{aligned}
& \left|f\left(\frac{a+b}{2}\right)-\frac{1}{b-a} \int_{a}^{b} f(x) d x\right| \leq \frac{(b-a)^{2}}{16} \int_{0}^{1} t^{2}\left[t^{s}\left|f^{\prime \prime}\left(\frac{a+b}{2}\right)\right|+(1-t)^{s}\left|f^{\prime \prime}(a)\right|-c t(1-t)(b-a)^{2}\right] d t \\
& +\frac{(b-a)^{2}}{16} \int_{0}^{1}(1-t)^{2}\left[t^{s}\left|f^{\prime \prime}(b)\right|+(1-t)^{s}\left|f^{\prime \prime}\left(\frac{a+b}{2}\right)\right|-c t(1-t)(b-a)^{2}\right] d t .
\end{aligned}
$$

Using the facts that

$$
\int_{0}^{1} t^{s+2} d t=\int_{0}^{1}(1-t)^{s+2}=\frac{1}{s+3}
$$

and

$$
\int_{0}^{1} t^{2}(1-t)^{s} d t=\int_{0}^{1} t^{s}(1-t)^{2} d t=\frac{2}{(s+1)(s+2)(s+3)}
$$

in (12), we obtain

$$
\begin{aligned}
& \left|f\left(\frac{a+b}{2}\right)-\frac{1}{b-a} \int_{a}^{b} f(x) d x\right| \leq \frac{(b-a)^{2}}{16}\left[\frac{1}{s+3}\left|f^{\prime \prime}\left(\frac{a+b}{2}\right)\right|+\frac{2\left|f^{\prime \prime}(a)\right|}{(s+1)(s+2)(s+3)}-\frac{c}{20}(b-a)^{2}\right] \\
& +\frac{(b-a)^{2}}{16}\left[\frac{2\left|f^{\prime \prime}(b)\right|}{(s+1)(s+2)(s+3)}+\frac{1}{s+3}\left|f^{\prime \prime}\left(\frac{a+b}{2}\right)\right|-\frac{c}{20}(b-a)^{2}\right] \\
& =\frac{(b-a)^{2}}{8[(s+1)(s+2)(s+3)]}\left\{\left|f^{\prime \prime}(a)\right|+\left|f^{\prime \prime}(b)\right|+(s+1)(s+2)\left|f^{\prime \prime}\left(\frac{a+b}{2}\right)\right|\right\}-\frac{c}{160}(b-a)^{2} .
\end{aligned}
$$

This completes the proof of the first inequality in (8).

For the proof of the second inequality in (8), since $\left|f^{\prime \prime}\right|$ is strongly s-convex, by (6), we have

$$
\left|f^{\prime \prime}\left(\frac{a+b}{2}\right)\right| \leq 2^{1-s}\left[\frac{\left|f^{\prime \prime}(a)\right|+\left|f^{\prime \prime}(b)\right|}{s+1}-\frac{c}{6}(b-a)^{2}\right]-\frac{c}{12}(b-a)^{2}
$$


Combining (15) and (16), we have

$$
\begin{aligned}
& \left|f\left(\frac{a+b}{2}\right)-\frac{1}{b-a} \int_{a}^{b} f(x) d x\right| \leq \frac{(b-a)^{2}}{8[(s+1)(s+2)(s+3)]} \\
& \times\left\{\left|f^{\prime \prime}(a)\right|+\left|f^{\prime \prime}(b)\right|+(s+1)(s+2)\left\{2^{1-s}\left[\frac{\left|f^{\prime \prime}(a)\right|+\left|f^{\prime \prime}(b)\right|}{s+1}-\frac{c}{6}(b-a)^{2}\right]-\frac{c}{12}(b-a)^{2}\right\}\right\}-\frac{c}{160}(b-a)^{2} \\
& =\frac{(b-a)^{2}}{8[(s+1)(s+2)(s+3)]} \times\left\{\left[1+(s+2) 2^{1-s}\right]\left[\left|f^{\prime \prime}(a)\right|+\left|f^{\prime \prime}(b)\right|\right]-\frac{\left[1+(s+1)(s+2) 2^{2-s}\right] c}{12}(b-a)^{2}\right\} \\
& -\frac{c}{160}(b-a)^{2}
\end{aligned}
$$

which completes the proof of the second inequality in (8), and thus the proof is completed.

Corollary 2. In Theorem 3, if we choose $s=1$, we have

$$
\begin{aligned}
\left|f\left(\frac{a+b}{2}\right)-\frac{1}{b-a} \int_{a}^{b} f(x) d x\right| & \leq \frac{(b-a)^{2}}{192}\left\{\left|f^{\prime \prime}(a)\right|+6\left|f^{\prime \prime}\left(\frac{a+b}{2}\right)\right|+\left|f^{\prime \prime}(b)\right|\right\}-\frac{c}{160}(b-a)^{2} \\
& \leq \frac{(b-a)^{2}}{48}\left[\left[\left|f^{\prime \prime}(a)\right|+\left|f^{\prime \prime}(b)\right|\right]\right]-\frac{13 c}{2304}(b-a)^{4}-\frac{c}{160}(b-a)^{2} .
\end{aligned}
$$

Theorem 4. Let $f: I \subset[0, \infty) \rightarrow \mathbb{R}$ be a twice differantiable mapping on $I^{0}$ such that $f^{\prime \prime} \in L[a, b]$, where a,b $\in I$ with $a<b$. If $\left|f^{\prime \prime}\right|^{q}$ is strongly s-convex on $[a, b]$ with modulus $c>0$, for some fixed $s \in(0,1]$, then the following inequality holds:

$$
\begin{aligned}
\left|f\left(\frac{a+b}{2}\right)-\frac{1}{b-a} \int_{a}^{b} f(x) d x\right| & \leq \frac{(b-a)^{2}}{16}\left(\frac{1}{2 p+1}\right)^{\frac{1}{p}}\left[\frac{1}{s+1}\left(\left|f^{\prime \prime}\left(\frac{a+b}{2}\right)\right|^{q}+\left|f^{\prime \prime}(a)\right|^{q}-\frac{c}{6}(b-a)^{2}\right)^{\frac{1}{q}}\right. \\
& \left.+\frac{1}{s+1}\left(\left|f^{\prime \prime}\left(\frac{a+b}{2}\right)\right|^{q}+\left|f^{\prime \prime}(b)\right|^{q}-\frac{c}{6}(b-a)^{2}\right)^{\frac{1}{q}}\right]
\end{aligned}
$$

where $q>1$ with $\frac{1}{p}+\frac{1}{q}=1$.

Proof. From Lemma 1 and using Hölder inequality, we have

$$
\begin{aligned}
& \left|f\left(\frac{a+b}{2}\right)-\frac{1}{b-a} \int_{a}^{b} f(t) d t\right| \leq \frac{(b-a)^{2}}{16}\left[\int_{0}^{1}|t|^{2}\left|f^{\prime \prime}\left(t \frac{a+b}{2}+(1-t) a\right)\right| d t+\int_{0}^{1}|t-1|^{2}\left|f^{\prime \prime}\left(t b+(1-t) \frac{a+b}{2}\right)\right| d t\right] \\
& \leq \frac{(b-a)^{2}}{16}\left[\left(\int_{0}^{1}|t|^{2 p} d t\right)^{\frac{1}{p}}\left(\int_{0}^{1}\left|f^{\prime \prime}\left(t \frac{a+b}{2}+(1-t) a\right)\right|^{q} d t\right)^{\frac{1}{q}}+\left(\int_{0}^{1}|t-1|^{2 p}\right)^{\frac{1}{p}}\left(\int_{0}^{1}\left|f^{\prime \prime}\left(t b+(1-t) \frac{a+b}{2}\right)\right|^{q} d t\right)^{\frac{1}{q}}\right] .
\end{aligned}
$$

Using strongly s-convexity of $\left|f^{\prime \prime}\right|^{q}$, we have

$$
\int_{0}^{1}\left|f^{\prime \prime}\left(t \frac{a+b}{2}+(1-t) a\right)\right|^{q} d t \leq \frac{1}{s+1}\left[\left|f^{\prime \prime}\left(\frac{a+b}{2}\right)\right|^{q}+\left|f^{\prime \prime}(a)\right|^{q}\right]-\frac{c}{6}(b-a)^{2}
$$


and

$$
\int_{0}^{1}\left|f^{\prime \prime}\left(t b+(1-t) \frac{a+b}{2}\right)\right|^{q} d t \leq \frac{1}{s+1}\left[\left|f^{\prime \prime}(b)\right|^{q}+\left|f^{\prime \prime}\left(\frac{a+b}{2}\right)\right|^{q}\right]-\frac{c}{6}(b-a)^{2} .
$$

By a simple computation, we have

$$
\int_{0}^{1} t^{2 p} d t=\int_{0}^{1}(1-t)^{2 p} d t=\frac{1}{2 p+1}
$$

If we put (20)-(22) in (19), we obtain

$$
\begin{aligned}
\left|f\left(\frac{a+b}{2}\right)-\frac{1}{b-a} \int_{a}^{b} f(t) d t\right| & \leq \frac{(b-a)^{2}}{16}\left(\frac{1}{2 p+1}\right)^{\frac{1}{p}}\left[\left(\frac{1}{s+1}\left[\left|f^{\prime \prime}\left(\frac{a+b}{2}\right)\right|^{q}+\left|f^{\prime \prime}(a)\right|^{q}\right]-\frac{c}{6}(b-a)^{2}\right)^{\frac{1}{q}}\right. \\
& \left.+\left(\frac{1}{s+1}\left[\left|f^{\prime \prime}(b)\right|^{q}+\left|f^{\prime \prime}\left(\frac{a+b}{2}\right)\right|^{q}\right]-\frac{c}{6}(b-a)^{2}\right)^{\frac{1}{q}}\right]
\end{aligned}
$$

which completes the proof.

Corollary 3. Under the assumption of Theorem 4 with $s=1$, we have

$$
\begin{aligned}
\left|f\left(\frac{a+b}{2}\right)-\frac{1}{b-a} \int_{a}^{b} f(x) d x\right| & \leq \frac{(b-a)^{2}}{16}\left(\frac{1}{2 p+1}\right)^{\frac{1}{p}}\left[\frac{1}{2}\left(\left|f^{\prime \prime}\left(\frac{a+b}{2}\right)\right|^{q}+\left|f^{\prime \prime}(a)\right|^{q}-\frac{c}{6}(b-a)^{2}\right)^{\frac{1}{q}}\right. \\
& \left.+\left(\frac{1}{2}\left[\left|f^{\prime \prime}\left(\frac{a+b}{2}\right)\right|^{q}+\left|f^{\prime \prime}(b)\right|^{q}\right]-\frac{c}{6}(b-a)^{2}\right)^{\frac{1}{q}}\right] .
\end{aligned}
$$

Theorem 5. Let $f: I \subset[0, \infty) \rightarrow \mathbb{R}$ be a twice differantiable mapping on $I^{0}$ such that $f^{\prime \prime} \in L[a, b]$, where $a, b \in I$ with $a<b$. If $\left|f^{\prime \prime}\right|^{q}$ is strongly s-convex on $[a, b]$ with modulus $c>0$, for some fixed $s \in(0,1]$, and $q \geq 1$, then the following inequality holds

$$
\begin{aligned}
& \left|f\left(\frac{a+b}{2}\right)-\frac{1}{b-a} \int_{a}^{b} f(x) d x\right| \leq \frac{(b-a)^{2}}{16}\left(\frac{1}{3}\right)^{\frac{1}{p}}\left[\left(\frac{1}{s+3}\left|f^{\prime \prime}\left(\frac{a+b}{2}\right)\right|^{q}+\frac{2}{(s+1)(s+2)(s+3)}\left|f^{\prime \prime}(a)\right|^{q}\right.\right. \\
& \left.\left.-\frac{c}{20}(b-a)^{2}\right)^{\frac{1}{q}}+\left(\frac{2}{(s+1)(s+2)(s+3)}\left|f^{\prime \prime}(b)\right|^{q}+\frac{1}{s+3}\left|f^{\prime \prime}\left(\frac{a+b}{2}\right)\right|^{q}-\frac{c}{20}(b-a)^{2}\right)^{\frac{1}{q}}\right] .
\end{aligned}
$$

Proof. From Lemma 1 and using the power mean inequality, we have

$$
\begin{aligned}
& \left|f\left(\frac{a+b}{2}\right)-\frac{1}{b-a} \int_{a}^{b} f(t) d t\right| \leq \frac{(b-a)^{2}}{16}\left[\int_{0}^{1} t^{2}\left|f^{\prime \prime}\left(t \frac{a+b}{2}+(1-t) a\right)\right|+\int_{0}^{1}(t-1)^{2}\left|f^{\prime \prime}\left(t b+(1-t) \frac{a+b}{2}\right)\right| d t\right] \\
& \leq \frac{(b-a)^{2}}{16}\left[\left(\int_{0}^{1} t^{2} d t\right)^{\frac{1}{p}}\left(\int_{0}^{1} t^{2}\left|f^{\prime \prime}\left(t \frac{a+b}{2}+(1-t) a\right)\right|^{q} d t\right)^{\frac{1}{q}}+\left(\int_{0}^{1}(t-1)^{2}\right)^{\frac{1}{p}}\left(\int_{0}^{1}(t-1)^{2}\left|f^{\prime \prime}\left(t b+(1-t) \frac{a+b}{2}\right)\right|^{q} d t\right)^{\frac{1}{q}}\right] .
\end{aligned}
$$


Since $\left|f^{\prime \prime}\right|^{q}$ is strongly $s$-convex, by a simple computations, we obtain

$$
\begin{aligned}
& \left|f\left(\frac{a+b}{2}\right)-\frac{1}{b-a} \int_{a}^{b} f(t) d t\right| \leq \frac{(b-a)^{2}}{16}\left[( \int _ { 0 } ^ { 1 } t ^ { 2 } d t ) ^ { \frac { 1 } { p } } \left(\int _ { 0 } ^ { 1 } t ^ { 2 } \left[t^{s}\left|f^{\prime \prime}\left(\frac{a+b}{2}\right)\right|^{q}\right.\right.\right. \\
& \left.\left.+(t-1)^{s}\left|f^{\prime \prime}(a)\right|^{q}-c t(1-t)(b-a)^{2}\right] d t\right)^{\frac{1}{q}} \\
& \left.+\left(\int_{0}^{1}(t-1)^{2}\right)^{\frac{1}{p}}\left(\int_{0}^{1}(t-1)^{2}\left[t^{s}\left|f^{\prime \prime}(b)\right|^{q}+(t-1)^{s}\left|f^{\prime \prime}\left(\frac{a+b}{2}\right)\right|^{q}-c t(1-t)(b-a)^{2}\right] d t\right)^{\frac{1}{q}}\right] \\
& \leq \frac{(b-a)^{2}}{16}\left(\frac{1}{3}\right)^{\frac{1}{p}}\left[\left(\frac{1}{s+3}\left|f^{\prime \prime}\left(\frac{a+b}{2}\right)\right|^{q}+\frac{2}{(s+1)(s+2)(s+3)}\left|f^{\prime \prime}(a)\right|^{q}-\frac{c}{20}(b-a)^{2}\right)^{\frac{1}{q}}\right. \\
& \left.+\left(\frac{2}{(s+1)(s+2)(s+3)}\left|f^{\prime \prime}(b)\right|^{q}+\frac{1}{s+3}\left|f^{\prime \prime}\left(\frac{a+b}{2}\right)\right|^{q}-\frac{c}{20}(b-a)^{2}\right)^{\frac{1}{q}}\right] .
\end{aligned}
$$

This completes the proof.

Corollary 4. In Theorem 5, if we choose $s=1$, we have

$$
\begin{aligned}
\left|f\left(\frac{a+b}{2}\right)-\frac{1}{b-a} \int_{a}^{b} f(x) d x\right| & \leq \frac{(b-a)^{2}}{48}\left(\frac{3}{4}\right)^{\frac{1}{q}}\left[\left(\left|f^{\prime \prime}\left(\frac{a+b}{2}\right)\right|^{q}+\frac{1}{3}\left|f^{\prime \prime}(a)\right|^{q}-\frac{c}{5}(b-a)^{2}\right)^{\frac{1}{q}}\right. \\
& \left.+\left(\frac{1}{3}\left|f^{\prime \prime}(b)\right|^{q}+\left|f^{\prime \prime}\left(\frac{a+b}{2}\right)\right|^{q}-\frac{c}{5}(b-a)^{2}\right)^{\frac{1}{q}}\right] .
\end{aligned}
$$

Now, we give the following Hadamard-type inequality for strongly $s$-concave mappings.

Theorem 6. Let $f: I \subset[0, \infty) \rightarrow \mathbb{R}$, be a twice differentiable mapping on $I^{0}$ such that $f^{\prime \prime} \in L[a, b]$, where a,b $\in I$ with $a<b$. If $\left|f^{\prime \prime}\right|^{q}$ is strongly s-concave on $[a, b]$ with the modulus $c>0$, for some fixed $s \in(0,1]$, then the following inequality holds

$$
\begin{aligned}
\left|f\left(\frac{a+b}{2}\right)-\frac{1}{b-a} \int_{a}^{b} f(t) d t\right| & \leq \frac{(b-a)^{2}}{16}\left(\frac{1}{2 p+1}\right)^{\frac{1}{p}} 2^{\frac{s-1}{q}}\left[\left.|| f^{\prime \prime}\left(\frac{3 a+b}{4}\right)\right|^{q}+\frac{c}{12}(b-a)^{2}\right]^{\frac{1}{q}} \\
& \left.+\left[\left|f^{\prime \prime}\left(\frac{a+3 b}{4}\right)\right|^{q}+\frac{c}{12}(b-a)^{2}\right]^{\frac{1}{q}}\right] .
\end{aligned}
$$

where $q>1$ and $\frac{1}{p}+\frac{1}{q}=1$.

Proof. From Lemma 1 and using Hölder inequality for $q>1$ and $\frac{1}{p}+\frac{1}{q}=1$, we obtain

$$
\begin{aligned}
\left|f\left(\frac{a+b}{2}\right)-\frac{1}{b-a} \int_{a}^{b} f(t) d t\right| & \leq \frac{(b-a)^{2}}{16}\left[\left(\int_{0}^{1}|t|^{2 p} d t\right)^{\frac{1}{p}}\left(\int_{0}^{1}\left|f^{\prime \prime}\left(t \frac{a+b}{2}+(1-t) a\right)\right|^{q} d t\right)^{\frac{1}{q}}\right. \\
& \left.+\left(\int_{0}^{1}|t-1|^{2 p}\right)^{\frac{1}{p}}\left(\int_{0}^{1}\left|f^{\prime \prime}\left(t b+(1-t) \frac{a+b}{2}\right)\right|^{q} d t\right)^{\frac{1}{q}}\right]
\end{aligned}
$$


Since $\left|f^{\prime \prime}\right|^{q}$ is strongly s-concave, we have

$$
\int_{0}^{1}\left|f^{\prime \prime}\left(t \frac{a+b}{2}+(1-t) a\right)\right|^{q} d t \leq 2^{s-1}\left[\left|f^{\prime \prime}\left(\frac{3 a+b}{4}\right)\right|^{q}+\frac{c}{12}(b-a)^{2}\right]
$$

and

$$
\int_{0}^{1}\left|f^{\prime \prime}\left(t b+(1-t) \frac{a+b}{2}\right)\right| d t \leq 2^{s-1}\left[\left|f^{\prime \prime}\left(\frac{a+3 b}{4}\right)\right|^{q}+\frac{c}{12}(b-a)^{2}\right] .
$$

From (27) and (28), we get

$$
\begin{aligned}
\left|f\left(\frac{a+b}{2}\right)-\frac{1}{b-a} \int_{a}^{b} f(t) d t\right| \leq & \frac{(b-a)^{2}}{16}\left(\frac{1}{2 p+1}\right)^{\frac{1}{p}} 2^{\frac{s-1}{q}}\left[\left[\left|f^{\prime \prime}\left(\frac{3 a+b}{4}\right)\right|^{q}+\frac{c}{12}(b-a)^{2}\right]^{\frac{1}{q}}\right. \\
& \left.+\left[\left|f^{\prime \prime}\left(\frac{a+3 b}{4}\right)\right|^{q}+\frac{c}{12}(b-a)^{2}\right]^{\frac{1}{q}}\right]
\end{aligned}
$$

which completes the proof.

Corollary 5. In theorem 6, we choose $s=1$ and $\frac{1}{3}<\left(\frac{1}{2 p+1}\right)^{\frac{1}{p}}<1, p>1$, we have

$$
\left|f\left(\frac{a+b}{2}\right)-\frac{1}{b-a} \int_{a}^{b} f(t) d t\right| \leq \frac{(b-a)^{2}}{16}\left[\left[\left|f^{\prime \prime}\left(\frac{3 a+b}{4}\right)\right|^{q}+\frac{c}{12}(b-a)^{2}\right]^{\frac{1}{q}}+\left[\left|f^{\prime \prime}\left(\frac{a+3 b}{4}\right)\right|^{q}+\frac{c}{12}(b-a)^{2}\right]^{\frac{1}{q}}\right] .
$$

\section{Concluding remarks}

In this study, using practical identity for twice differentiable functions proved by Özdemir et al., we present some new upper bounds for Midpoint type inequalities by taking advantageous of mappings whose second derivatives in absolute values are strongly $s$-convex.

\section{Competing interests}

The authors declare that they have no competing interests.

\section{Authors' contributions}

All authors have contributed to all parts of the article. All authors read and approved the final manuscript.

\section{References}

[1] H. Angulo, J. Gimenez, A. M. Moros, and K. Nikodem, On strongly h-convex function, Ann. Funct. Anal. 2(2), $2011,85-91$.

[2] M. K. Bakula and J. Pečarić, Note on some Hadamard-type inequalities, Journal of Inequalities in Pure and Applied Mathematics, vol. 5, no. 3, article 74, 2004. 
[3] S. S. Dragomir and C. E. M. Pearce, Selected Topics on Hermite-Hadamard Inequalities and Applications, RGMIA Monographs, Victoria University, 2000.

[4] S. S. Dragomir and R.P. Agarwal, Two inequalities for differentiable mappings and applications to special means of real numbers and to trapezoidal formula, Appl. Math. lett., 11(5) (1998), 91-95.

[5] H. Hudzik, L. Maligranda, Some remarks on s-convex functions. Aequ. Math. 48, 100-111 (1994).

[6] J. Makó and A. Házy, On strongly convex functions. Carpathian Journal of Mathematics, 32 (1). 87-95.

[7] N. Merentes, K. Nikodem, Remarks on strongly convex functions, Aequationes Math. 80 (2010) 193-199.

[8] K. Nikodem, Z. Pales, Characterizations of inner product spaces be strongly convex functions, Banach J. Math. Anal. 5 (2011) 83-87.

[9] M. E. Özdemir, Ç. Yıldız, A. O. Akdemir and E. Set, On some inequalities for s-convex functions and applications, Journal of Inequalities and Applications 2013, 2013:333.

[10] J.E. Pečarić, F. Proschan and Y.L. Tong, Convex functions, partial orderings and statistical applications, Academic Press, Boston, 1992.

[11] B.T. Polyak, Existence theorems and convergence of minimizing sequences in extremum problems with restictions, Soviet Math. Dokl. 7 (1966), 72-75.

[12] M. Z. Sarikaya, On strongly $\varphi_{h}$-convex functions in inner product spaces, Arabian Journal of Mathematics, (2013) 2:295-302.

[13] M. Z. Sarikaya, E. Set, M. E. Ozdemir and S. S. Dragomir, New some Hadamard's type inequalities for co-ordinated convex functions, Tamsui Oxford Journal of Information and Mathematical Sciences, 28(2) (2012) 137-152.

[14] M. Z. Sarikaya and H. Yaldiz, On Hermite Hadamard-type inequalities for strongly log-convex functions, International Journal of Modern Mathematical Sciences, 2013, 5(3): 92-98.

[15] M. Z. Sarikaya and H. Yaldiz, On the Hadamard's type inequalities for L-Lipschitzian mapping, Konuralp Journal of Mathematics, $1(2), 33-40$ (2013)

[16] M. Z. Sarikaya and K. Ozcelik, On Hermite-Hadamard type integral inequalities for strongly $\varphi_{h}-$ convex functions, International Journal of Advanced Research in Engineering and Applied Sciences (IJAREAS), 1(1), pp:34-52, 2014.

[17] M. Z. Sarikaya, On Hermite Hadamard-type inequalities for strongly $\varphi$-convex functions, Southeast Asian Bull. Math., 39(1) (2015) , pp: 123-132. 